\title{
On characterizations of Bloch spaces and Besov spaces of pluriharmonic mappings
}

$\mathrm{Xi} \mathrm{Fu}{ }^{1}$ and Xiaoyou Liur2*

"Correspondence:
liuxiaoyou2002@hotmail.com
${ }^{2}$ School of Mathematics and
Physics, University of South China,
Hengyang, Hunan 421001, People's
Republic of China
Full list of author information is
available at the end of the article

available at the end of the article

\begin{abstract}
We characterize the Bloch spaces and Besov spaces of pluriharmonic mappings on the unit ball of $\mathbb{C}^{n}$ by using the following quantity:

$\sup _{\rho(z, w)<r, z \neq w} \frac{\left(1-|z|^{2}\right)^{\alpha}\left(1-|w|^{2}\right)^{\beta}\left|\hat{D}^{(m)} f(z)-\hat{D}^{(m)} f(w)\right|}{|z-w|}$, where $\alpha+\beta=n+1, \hat{D}^{(m)}=\frac{\partial^{m}}{\partial z^{m}}+\frac{\partial^{m}}{\partial \bar{z}^{m}}$, $|m|=n$. This generalizes the main results of (Yoneda in Proc. Edinb. Math. Soc. 45:229-239, 2002) in the higher dimensional case.
\end{abstract}

MSC: Primary 30C65; 30C45; secondary 30C20

Keywords: pluriharmonic mapping; Bloch space; Besov space

\section{Introduction}

Let $\mathbb{C}^{n}=\left\{z=\left(z_{1}, \ldots, z_{n}\right): z_{1}, \ldots, z_{n} \in \mathbb{C}\right\}$ denote the $n$ dimensional complex vector space. For $a=\left(a_{1}, \ldots, a_{n}\right) \in \mathbb{C}^{n}$, we define the Euclidean inner product $\langle\cdot, \cdot\rangle$ by

$$
\langle z, a\rangle=z_{1} \bar{a}_{1}+\cdots+z_{n} \bar{a}_{n}
$$

where $\bar{a}_{k}(k \in\{1, \ldots, n\})$ denotes the complex conjugate of $a_{k}$. Then the Euclidean length of $z$ is defined by

$$
|z|=\langle z, z\rangle^{\frac{1}{2}}=\left(\left|z_{1}\right|^{2}+\cdots+\left|z_{n}\right|^{2}\right)^{\frac{1}{2}} .
$$

Denote a ball in $\mathbb{C}^{n}$ with center $a$ and radius $r>0$ by

$$
\mathbb{B}^{n}(a, r)=\left\{z \in \mathbb{C}^{n}:|z-a|<r\right\} .
$$

In particular, we let $\mathbb{B}^{n}$ denote the unit ball $\mathbb{B}^{n}(0,1)$ and let $\mathbb{D}$ be the unit disk in $\mathbb{C}$.

A complex-valued function $f$ of $\mathbb{B}^{n}$ into $\mathbb{C}$ is called pluriharmonic if there are two holomorphic functions $h$ and $g$, such that $f=h+\bar{g}$. We denote by $\mathcal{P}\left(\mathbb{B}^{n}\right)$ the class of all pluriharmonic mappings on the unit ball of $\mathbb{C}^{n}$.

Let $f=h+\bar{g} \in \mathcal{P}\left(\mathbb{B}^{n}\right)$. For a multi-index $m=\left(m_{1}, \ldots, m_{n}\right)$, we employ the notations

$$
\begin{aligned}
& \nabla f(z)=\left(\frac{\partial f}{\partial z_{1}}, \ldots, \frac{\partial f}{\partial z_{n}}\right), \quad \bar{\nabla} f(z)=\left(\frac{\partial f}{\partial \bar{z}_{1}}, \ldots, \frac{\partial f}{\partial \bar{z}_{n}}\right), \\
& \partial^{m} f=\frac{\partial^{m} f}{\partial z^{m}}=\frac{\partial^{|m|} f}{\partial z_{1}^{m_{1}} \cdots \partial z_{n}^{m_{n}}}, \quad \bar{\partial}^{m} f=\frac{\partial^{m} f}{\partial \bar{z}^{m}}=\frac{\partial^{|m|} f}{\partial \bar{z}_{1}^{m_{1}} \cdots \partial \bar{z}_{n}^{m_{n}}},
\end{aligned}
$$

(c) $2015 \mathrm{Fu}$ and Liu. This article is distributed under the terms of the Creative Commons Attribution 4.0 International License (http://creativecommons.org/licenses/by/4.0/), which permits unrestricted use, distribution, and reproduction in any medium, provided you give appropriate credit to the original author(s) and the source, provide a link to the Creative Commons license, and indicate if changes were made. 


$$
\hat{D}^{(m)} f=\partial^{m} f+\bar{\partial}^{m} f=\partial^{m} h+\bar{\partial}^{m} g
$$

where $|m|=m_{1}+\cdots+m_{n}$. Obviously, if $f \in \mathcal{P}\left(\mathbb{B}^{n}\right)$, then so does $\hat{D}^{(m)} f$.

Similar to the planar case, the Bloch space $\mathcal{P} \mathcal{B}\left(\mathbb{B}^{n}\right)$ of $\mathcal{P}\left(\mathbb{B}^{n}\right)$ consists of all mappings $f \in \mathcal{P}\left(\mathbb{B}^{n}\right)$ such that

$$
\|f\|=\sup _{z \in \mathbb{B}^{n}}\left(1-|z|^{2}\right)(|\nabla f(z)|+|\bar{\nabla} f(z)|)<\infty
$$

the little Bloch space $\mathcal{P} \mathcal{B}_{0}\left(\mathbb{B}^{n}\right)$ consists of all mappings $f \in \mathcal{P B}\left(\mathbb{B}^{n}\right)$ such that

$$
\lim _{|z| \rightarrow 1^{-}}\left(1-|z|^{2}\right)(|\nabla f(z)|+|\bar{\nabla} f(z)|)=0 .
$$

Let $d \lambda(z)=\left(1-|z|^{2}\right)^{-n-1} d v(z)$, where $d v$ is the normalized Lebesgue measure of $\mathbb{B}^{n}$. For $1 \leq p<\infty$, the Besov space $\mathcal{B}_{p}$ of $\mathcal{P}\left(\mathbb{B}^{n}\right)$ consists of all mappings $f \in \mathcal{P}\left(\mathbb{B}^{n}\right)$ such that $(1-$ $\left.|z|^{2}\right)(|\nabla f(z)|+|\bar{\nabla} f(z)|) \in L^{p}\left(\mathbb{B}^{n}, d \lambda\right)$, i.e.

$$
\|f\|_{L^{p}(d \lambda(z))}=\int_{\mathbb{B}^{n}}\left(\left(1-|z|^{2}\right)(|\nabla f(z)|+|\bar{\nabla} f(z)|)\right)^{p} d \lambda(z)<\infty
$$

For a planar harmonic mapping $f$ in $\mathbb{D}$, Colonna [1] proved that $f \in \mathcal{P B}(\mathbb{D})$ if and only if the Lipschitz number

$$
\beta_{f}=\sup _{z, w \in \mathbb{D}, z \neq w} \frac{|f(z)-f(w)|}{\operatorname{arctanh}\left|\frac{z-w}{1-\bar{z} w}\right|}<\infty
$$

Let

$$
l=\sup _{w \in D(z, r), z \neq w} \frac{\left(1-|z|^{2}\right)^{\alpha}\left(1-|w|^{2}\right)^{\beta}\left|\hat{D}^{(n-1)} f(z)-\hat{D}^{(n-1)} f(w)\right|}{|z-w|},
$$

where $D(z, r)$ is the Bergman disc with center $z \in \mathbb{D}$ and radius $r, n \geq 1$ an integer and $\alpha+\beta=n$. By means of it, Yoneda [2] characterized the spaces $\mathcal{P} \mathcal{B}(\mathbb{D})$ and $\mathcal{B}_{p}$ as follows.

Theorem $\mathbf{A}$ Let $n \geq 1$ be an integer and $f \in \mathcal{P}(\mathbb{D})$. Then $f \in \mathcal{P B}(\mathbb{D})$ if and only if $l$ is bounded.

Theorem B Let $n \geq 1$ be an integer and $f \in \mathcal{P}(\mathbb{D})$. Then $f \in \mathcal{B}_{p}$ if and only if

$$
\int_{\mathbb{D}} l^{p} d \lambda(z)<\infty
$$

In this article, we consider the corresponding problems in higher dimensional setting. We refer to [3-7] for the related topics for holomorphic or harmonic functions. See [8-12] for various characterizations of the Bloch, little Bloch, and Besov spaces in the unit ball of $\mathbb{C}^{n}$. In Section 2, we recall some basic facts for pluriharmonic mappings. Our main results are Theorems 1-4, whose proofs will be presented in Sections 3 and 4. 


\section{Preliminaries}

Let $\operatorname{Aut}\left(\mathbb{B}^{n}\right)$ denote the group of biholomorphic mappings of $\mathbb{B}^{n}$ onto itself. It is well known that $\operatorname{Aut}\left(\mathbb{B}^{n}\right)$ is generated by the unitary operators on $\mathbb{B}^{n}$ and the involutions $\phi_{a}$ of the form

$$
\phi_{a}(z)=\frac{a-P_{a} z-\left(1-|a|^{2}\right)^{\frac{1}{2}} Q_{a} z}{1-\langle z, a\rangle},
$$

where $a, z \in \mathbb{B}^{n}$,

$$
P_{a} z=\frac{a\langle z, a\rangle}{\langle a, a\rangle}, \quad Q_{a} z=z-P_{a} z
$$

For $z$, w $\in \mathbb{B}^{n}$, we define $\rho(z, w)=\left|\phi_{z}(w)\right|$. It is known that $\rho$ is a distance function on $\mathbb{B}^{n}$, and we call it pseudo-hyperbolic metric $(c f$. $[6,12])$. For $r \in(0,1)$, the pseudo-hyperbolic ball with center $z$ and radius $r$ is given by

$$
E(z, r)=\left\{w \in \mathbb{B}:\left|\phi_{z}(w)\right|<r\right\} .
$$

Clearly, $E(z, r)=\phi_{z}(\mathbb{B}(0, r))$.

Lemma 1 ([12]) Let $0<r<1$ and $w \in E(z, r)$. Then

$$
1-|z|^{2} \asymp 1-|w|^{2} \asymp|1-\langle z, w\rangle| \asymp|E(z, r)|^{\frac{1}{n+1}},
$$

where $|E(z, r)|$ is the normalized volume of $E(z, r), A \asymp B$ means that there is a constant $C>0$ such that $B / C \leq A \leq B C$.

The following lemma is crucial [13].

Lemma 2 Suppose that $f: \overline{\mathbb{B}}^{n}(a, r) \rightarrow \mathbb{C}$ is continuous and pluriharmonic in $\mathbb{B}^{n}(a, r)$. Then there exists $C>0$ such that

$$
|\nabla f(a)|+|\bar{\nabla} f(a)| \leq \frac{C}{r} \int_{\partial \mathbb{B}^{n}}|f(a+r \zeta)-f(a)| d \sigma(\zeta) .
$$

Let $h$ be a holomorphic function in $\mathbb{B}^{n}$. We say that $h \in \mathcal{B}$ if

$$
\sup _{z \in \mathbb{B}^{n}}\left(1-|z|^{2}\right)|\nabla h(z)|<\infty
$$

similarly, $h \in \mathcal{B}_{0}$ if $h \in \mathcal{B}$ and

$$
\lim _{|z| \rightarrow 1^{-}}\left(1-|z|^{2}\right)|\nabla h(z)|=0
$$

It is obvious that a pluriharmonic mapping $f=h+\bar{g} \in \mathcal{P}\left(\mathbb{B}^{n}\right)\left(\right.$ resp. $\left.\mathcal{P} \mathcal{B}_{0}\left(\mathbb{B}^{n}\right)\right)$ if and only if both $h, g \in \mathcal{B}$ (resp. $\mathcal{B}_{0}$ ).

The following is a characterization of the space $\mathcal{B}\left(\right.$ resp. $\left.\mathcal{B}_{0}\right)$. 
Lemma 3 ([12]) Let $h$ be holomorphic in $\mathbb{B}^{n}$ and $N$ a positive integer. Then $h \in \mathcal{B}$ (resp. $\mathcal{B}_{0}$ ) if and only if

$$
\sup _{z \in \mathbb{B}^{n}}\left(1-|z|^{2}\right)^{N}\left|\frac{\partial^{m} h(z)}{\partial z^{m}}\right|<\infty \quad\left(\text { resp. } \lim _{|z| \rightarrow 1^{-}}\left(1-|z|^{2}\right)^{N}\left|\frac{\partial^{m} h(z)}{\partial z^{m}}\right|=0\right)
$$

for all values of the multi-index $m$ with $|m|=N$.

Corollary 1 Let $f=h+\bar{g}$ be a pluriharmonic mapping in $\mathbb{B}^{n}$ and $N$ a positive integer. Then $f \in \mathcal{P B}\left(\mathbb{B}^{n}\right)\left(\right.$ resp. $\left.\mathcal{P} \mathcal{B}_{0}\left(\mathbb{B}^{n}\right)\right)$ if and only if

$$
\sup _{z \in \mathbb{B}^{n}}\left(1-|z|^{2}\right)^{N}\left(\left|\partial^{m} f\right|+\left|\bar{\partial}^{m} f\right|\right)=\sup _{z \in \mathbb{B}^{n}}\left(1-|z|^{2}\right)^{N}\left(\left|\partial^{m} h\right|+\left|\bar{\partial}^{m} g\right|\right)<\infty,
$$

respectively,

$$
\lim _{|z| \rightarrow 1^{-}}\left(1-|z|^{2}\right)^{N}\left(\left|\partial^{m} f\right|+\left|\bar{\partial}^{m} f\right|\right)=\lim _{|z| \rightarrow 1^{-}}\left(1-|z|^{2}\right)^{N}\left(\left|\partial^{m} h\right|+\left|\bar{\partial}^{m} g\right|\right) \rightarrow 0
$$

for all values of the multi-index $m$ with $|m|=N$.

As an application of Lemma 3, we obtain the following.

Lemma 4 Let $h$ be holomorphic in $\mathbb{B}^{n}$. Then $h \in \mathcal{B}$ if and only iffor each $j \in\{1, \ldots, n\}$,

$$
L=\sup _{z, w \in \mathbb{B}^{n}, z \neq w} \frac{\left(1-|z|^{2}\right)\left(1-|w|^{2}\right)}{|z-w|}\left|\frac{\partial h(z)}{\partial z_{j}}-\frac{\partial h(w)}{\partial z_{j}}\right|<\infty .
$$

Proof Fixing a point $w$ and letting

$$
z=w+\overline{\xi \nabla\left(\frac{\partial h}{\partial z_{j}}\right)(w)} \rightarrow w
$$

with $\xi \in \mathbb{C}$, we have

$$
\left(1-|w|^{2}\right)^{2}\left|\nabla\left(\frac{\partial h}{\partial z_{j}}\right)(w)\right| \leq L
$$

for each $j \in\{1, \ldots, n\}$. By Lemma 3 , we see that $h \in \mathcal{B}$.

For the converse, we assume that $h \in \mathcal{B}$. Let $h_{j}(z)=\frac{\partial h(z)}{\partial z_{j}}$, then for each $j \in\{1, \ldots, n\}$,

$$
\begin{aligned}
\left|h_{j}(z)-h_{j}(w)\right| & =\left|\int_{0}^{1} \frac{d h_{j}}{d s}(s z+(1-s) w) d s\right| \\
& \leq \sum_{k=1}^{n}\left|\left(z_{k}-w_{k}\right) \int_{0}^{1} \frac{\partial h_{j}}{\partial z_{k}}(s z+(1-s) w) d s\right| \\
& \leq \sqrt{n}|z-w| \int_{0}^{1}\left|\nabla h_{j}(s z+(1-s) w)\right| d s \\
& \leq C|z-w| \int_{0}^{1} \frac{d s}{\left(1-|s z+(1-s) w|^{2}\right)^{2}} .
\end{aligned}
$$


It follows from [7] that there exists $0<C_{1}<\infty$ such that

$$
\int_{0}^{1} \frac{d s}{\left(1-|s z+(1-s) w|^{2}\right)^{2}} \leq \frac{C_{1}}{\left(1-|z|^{2}\right)\left(1-|w|^{2}\right)}
$$

This implies that

$$
\sup _{z, w \in \mathbb{B}^{n}, z \neq w} \frac{\left(1-|z|^{2}\right)\left(1-|w|^{2}\right)}{|z-w|}\left|h_{j}(z)-h_{j}(w)\right|<\infty .
$$

So the result follows.

\section{The Bloch space for pluriharmonic mappings}

In this section, we give some characterizations of the spaces $\mathcal{P B}\left(\mathbb{B}^{n}\right)$ and $\mathcal{P} \mathcal{B}_{0}\left(\mathbb{B}^{n}\right)$ which can be viewed as the generalizations of Yoneda's results in the higher dimensional case.

Theorem 1 Let $f \in \mathcal{P}\left(\mathbb{B}^{n}\right), N \geq 0$ be an integer and $0<r<1$. Then $f \in \mathcal{P B}\left(\mathbb{B}^{n}\right)$ if and only if

$$
L_{f}=\sup _{z \in \mathbb{B}^{n}, \rho(z, w)<r, z \neq w} \frac{\left(1-|z|^{2}\right)^{\alpha}\left(1-|w|^{2}\right)^{\beta}\left|\hat{D}^{(m)} f(z)-\hat{D}^{(m)} f(w)\right|}{|z-w|}<\infty
$$

for all values of the multi-index $m$ with $|m|=N$, where $\alpha+\beta=N+1$.

Proof First we prove the sufficiency. Let $f(z) \in \mathcal{P}\left(\mathbb{B}^{n}\right)$, then for each multi-index $m$ with $|m|=N, \hat{D}^{(m)} f(z)$ is also pluriharmonic. According to Lemma 2 , for $z \in \mathbb{B}^{n}$ and $r \in(0,1)$,

$$
\left|\nabla\left(\hat{D}^{(m)} f\right)(z)\right|+\left|\bar{\nabla}\left(\hat{D}^{(m)} f\right)(z)\right| \leq \frac{C}{\left(1-|z|^{2}\right)} \int_{\partial \mathbb{B}^{n}}\left|\left(\hat{D}^{(m)} f\right)(z+\varrho \zeta)-\left(\hat{D}^{(m)} f\right)(z)\right| d \sigma(\zeta),
$$

where $\varrho=\frac{r\left(1-|z|^{2}\right)}{2}$. By a simple computation, we see that $\mathbb{B}^{n}(z, \varrho) \subset E(z, r)$, so

$$
\left|\nabla\left(\hat{D}^{(m)} f\right)(z)\right|+\left|\bar{\nabla}\left(\hat{D}^{(m)} f\right)(z)\right| \leq \frac{C}{\left(1-|z|^{2}\right)} \sup _{w \in E(z, r)}\left|\left(\hat{D}^{(m)} f\right)(z)-\left(\hat{D}^{(m)} f\right)(w)\right| .
$$

Since for each $w \in E(z, r), w \neq z$,

$$
\frac{\left(1-|z|^{2}\right)^{\frac{1}{2}}\left(1-|w|^{2}\right)^{\frac{1}{2}}}{|z-w|} \geq \frac{\left(1-r^{2}\right)^{\frac{1}{2}}}{r},
$$

by Lemma 1, we can deduce that

$$
\frac{\left(1-|z|^{2}\right)^{\alpha}\left(1-|w|^{2}\right)^{\beta}}{|z-w|} \geq C_{1}\left(1-|z|^{2}\right)^{N} .
$$

Therefore, there exists a positive constant $C_{2}$ such that

$$
\left(1-|z|^{2}\right)^{N+1}\left(\left|\nabla\left(\hat{D}^{(m)} f\right)\right|+\left|\bar{\nabla}\left(\hat{D}^{(m)} f\right)\right|\right) \leq C_{2} L_{f},
$$

from which we see that $f \in \mathcal{P} \mathcal{B}\left(\mathbb{B}^{n}\right)$. 
Now we prove the necessity. Let $w \in E(z, r), w \neq z$. Then for each multi-index $m$ with $|m|=N$, we have

$$
\begin{aligned}
\left|\left(\hat{D}^{(m)} f\right)(z)-\left(\hat{D}^{(m)} f\right)(w)\right|= & \left|\int_{0}^{1} \frac{d\left(\hat{D}^{(m)} f\right)}{d s}(s z+(1-s) w) d s\right| \\
\leq & \sum_{k=1}^{n}\left|\left(z_{k}-w_{k}\right) \int_{0}^{1} \frac{\partial\left(\hat{D}^{(m)} f\right)}{\partial z_{k}}(s z+(1-s) w) d s\right| \\
& +\sum_{k=1}^{n}\left|\left(\bar{z}_{k}-\bar{w}_{k}\right) \int_{0}^{1} \frac{\partial\left(\hat{D}^{(m)} f\right)}{\partial \bar{z}_{k}}(s z+(1-s) w) d s\right| \\
\leq & \sqrt{n}|z-w| \int_{0}^{1}\left(\left|\nabla\left(\hat{D}^{(m)} f\right)(s z+(1-s) w)\right|\right. \\
& \left.+\left|\bar{\nabla}\left(\hat{D}^{(m)} f\right)(s z+(1-s) w)\right|\right) d s \\
\leq & C|z-w| \int_{0}^{1} \frac{d s}{(1-|s z+(1-s) w|)^{N+1}} .
\end{aligned}
$$

By Lemma 1 we infer that there exists $\iota>0$ such that $1-|w|=\iota(1-|z|)$ and

$$
\begin{aligned}
\frac{\left|\left(\hat{D}^{(m)} f\right)(z)-\left(\hat{D}^{(m)} f\right)(w)\right|}{|z-w|} & \leq C \int_{0}^{1} \frac{d s}{(s(1-|z|)+(1-s)(1-|w|))^{N+1}} \\
& \leq \frac{C^{\prime}}{\left(1-|z|^{2}\right)^{N+1}} \int_{0}^{1} \frac{d s}{[s+\iota(1-s)]^{N+1}} \\
& \leq \frac{C^{\prime \prime}}{\left(1-|z|^{2}\right)^{\alpha}\left(1-|w|^{2}\right)^{\beta}} .
\end{aligned}
$$

Thus,

$$
L_{f}=\sup _{z \in \mathbb{B}^{n}, \rho(z, w)<r, z \neq w} \frac{\left(1-|z|^{2}\right)^{\alpha}\left(1-|w|^{2}\right)^{\beta}\left|\hat{D}^{(m)} f(z)-\hat{D}^{(m)} f(w)\right|}{|z-w|}<\infty .
$$

So the proof is complete.

Theorem 2 Let $f \in \mathcal{P}\left(\mathbb{B}^{n}\right)$ and $N=1,2$. Then $f \in \mathcal{P B}\left(\mathbb{B}^{n}\right)$ if and only if

$$
\sup _{z, w \in \mathbb{B}^{n}, z \neq w}\left(1-|z|^{2}\right)^{\frac{N}{2}}\left(1-|w|^{2}\right)^{\frac{N}{2}}\left|\frac{\left(\hat{D}^{(m)} f\right)(z)-\left(\hat{D}^{(m)} f\right)(w)}{z-w}\right|<\infty
$$

for all multi-index with $|m|=N-1$.

Proof The sufficiency follows from Theorem 1 . We only need to prove the necessity. When $N=1$, we refer to $[8,11]$. Now we prove $N=2$. Let $f=h+\bar{g}$. Then for each $j \in\{1, \ldots, n\}$,

$$
\begin{aligned}
& \sup _{z, w \in \mathbb{B}^{n}, z \neq w} \frac{\left(1-|z|^{2}\right)\left(1-|w|^{2}\right)}{|z-w|}\left|\frac{\partial f(z)}{\partial z_{j}}+\frac{\partial f(z)}{\partial \bar{z}_{j}}-\frac{\partial f(w)}{\partial z_{j}}-\frac{\partial f(w)}{\partial \bar{z}_{j}}\right| \\
& \leq \sup _{z, w \in \mathbb{B}^{n}, z \neq w} \frac{\left(1-|z|^{2}\right)\left(1-|w|^{2}\right)}{|z-w|}\left(\left|\frac{\partial h(z)}{\partial z_{j}}-\frac{\partial h(w)}{\partial z_{j}}\right|+\left|\frac{\partial g(z)}{\partial z_{j}}-\frac{\partial g(w)}{\partial z_{j}}\right|\right) .
\end{aligned}
$$


Since $f \in \mathcal{P B}\left(\mathbb{B}^{n}\right), h, g \in \mathcal{B}$, by Lemma 4 ,

$$
\begin{aligned}
& \sup _{z, w \in \mathbb{B}^{n}, z \neq w} \frac{\left(1-|z|^{2}\right)\left(1-|w|^{2}\right)}{|z-w|}\left|\frac{\partial h(z)}{\partial z_{j}}-\frac{\partial h(w)}{\partial z_{j}}\right|<\infty, \\
& \leq \sup _{z, w \in \mathbb{B}^{n}, z \neq w} \frac{\left(1-|z|^{2}\right)\left(1-|w|^{2}\right)}{|z-w|}\left|\frac{\partial g(z)}{\partial z_{j}}-\frac{\partial g(w)}{\partial z_{j}}\right|<\infty .
\end{aligned}
$$

This completes the proof.

Theorem 3 Let $f \in \mathcal{P B}\left(\mathbb{B}^{n}\right), N \geq 0$ be an integer and $0<r<1$. Then $f \in \mathcal{P} \mathcal{B}_{0}\left(\mathbb{B}^{n}\right)$ if and only if

$$
\lim _{|z| \rightarrow 1^{-}} \sup _{z \in \mathbb{B}^{n}, \rho(z, w)<r, z \neq w} \frac{\left(1-|z|^{2}\right)^{\alpha}\left(1-|w|^{2}\right)^{\beta}\left|\hat{D}^{(m)} f(z)-\hat{D}^{(m)} f(w)\right|}{|z-w|}=0
$$

for all values of the multi-index $m$ with $|m|=N$, where $\alpha+\beta=N+1$.

Proof Sufficiency. Assume that (1) holds. Then for any $\epsilon>0$, there exists $\delta \in(0,1)$ such that

$$
\sup _{z \in \mathbb{B}^{n}, \rho(z, w)<r, z \neq w} \frac{\left(1-|z|^{2}\right)^{\alpha}\left(1-|w|^{2}\right)^{\beta}\left|\hat{D}^{(m)} f(z)-\hat{D}^{(m)} f(w)\right|}{|z-w|}<\epsilon
$$

whenever $\delta<|z|<1$. It follows from an argument similar to the proof of Theorem 1, that we have

$$
\begin{aligned}
& \left(1-|z|^{2}\right)^{N+1}\left(\left|\nabla\left(\hat{D}^{(m)} f\right)\right|+\left|\bar{\nabla}\left(\hat{D}^{(m)} f\right)\right|\right) \\
& \quad \leq C \sup _{z \in \mathbb{B}^{n}, \rho(z, w)<r, z \neq w} \frac{\left(1-|z|^{2}\right)^{\alpha}\left(1-|w|^{2}\right)^{\beta}\left|\hat{D}^{(m)} f(z)-\hat{D}^{(m)} f(w)\right|}{|z-w|}<C \epsilon,
\end{aligned}
$$

whenever $\delta<|z|<1$. Hence

$$
\lim _{|z| \rightarrow 1^{-}}\left(1-|z|^{2}\right)^{N+1}\left(\left|\nabla\left(\hat{D}^{(m)} f\right)\right|+\left|\bar{\nabla}\left(\hat{D}^{(m)} f\right)\right|\right)=0,
$$

from which we see that $f \in \mathcal{P} \mathcal{B}_{0}\left(\mathbb{B}^{n}\right)$.

Necessity. For $\lambda \in(0,1)$, let $f_{\lambda}(z)=f(\lambda z)$. By Lemma 1 and the proof of Theorem 1 , we see that for each multi-index $m$ with $|m|=N$,

$$
\begin{aligned}
& \frac{\left(1-|z|^{2}\right)^{\alpha}\left(1-|w|^{2}\right)^{\beta}\left|\hat{D}^{(m)}\left(f-f_{\lambda}\right)(z)-\hat{D}^{(m)}\left(f-f_{\lambda}\right)(w)\right|}{|z-w|} \\
& \quad \leq C_{1}\left(1-|\xi|^{2}\right)^{N+1}\left(\left|\nabla \hat{D}^{(m)}\left(f-f_{\lambda}\right)(\xi)\right|+\left|\bar{\nabla} \hat{D}^{(m)}\left(f-f_{\lambda}\right)(\xi)\right|\right)
\end{aligned}
$$

and

$$
\begin{aligned}
& \frac{\left(1-|z|^{2}\right)^{\alpha}\left(1-|w|^{2}\right)^{\beta}\left|\hat{D}^{(m)} f_{\lambda}(z)-\hat{D}^{(m)} f_{\lambda}(w)\right|}{|z-w|} \\
& \quad \leq \frac{C_{2} \lambda}{\left(1-|\lambda|^{2}\right)^{N+1}}\left(1-|\eta|^{2}\right)^{N+1}\left(\left|\nabla\left(\hat{D}^{(m)} f_{\lambda}\right)(\eta)\right|+\left|\bar{\nabla}\left(\hat{D}^{(m)} f_{\lambda}\right)(\eta)\right|\right)
\end{aligned}
$$


for all $z, w \in \mathbb{B}^{n}, \rho(z, w)<r$ and $\xi, \eta \in E(z, r)$. So

$$
\begin{aligned}
L_{f} \leq & C_{1}\left(1-|\xi|^{2}\right)^{N+1}\left(\left|\nabla \hat{D}^{(m)}\left(f-f_{\lambda}\right)(\xi)\right|+\left|\bar{\nabla} \hat{D}^{(m)}\left(f-f_{\lambda}\right)(\xi)\right|\right) \\
& +\frac{C_{2} \lambda}{\left(1-|\lambda|^{2}\right)^{N+1}}\left(1-|\eta|^{2}\right)^{N+1}\left(\left|\nabla\left(\hat{D}^{(m)} f_{\lambda}\right)(\eta)\right|+\left|\bar{\nabla}\left(\hat{D}^{(m)} f_{\lambda}\right)(\eta)\right|\right) .
\end{aligned}
$$

First letting $|z| \rightarrow 1^{-}$and then letting $\lambda \rightarrow 1^{-}$, we obtain the desired result.

From Theorem 2 and the proof of Theorem 3, we have the following.

Corollary 2 Let $f \in \mathcal{P B}\left(\mathbb{B}^{n}\right)$ and $N=1,2$. Then $f \in \mathcal{P} \mathcal{B}_{0}\left(\mathbb{B}^{n}\right)$ if and only if

$$
\lim _{|z| \rightarrow 1^{-}} \sup _{z, w \in \mathbb{B}^{n}, z \neq w}\left(1-|z|^{2}\right)^{\frac{N}{2}}\left(1-|w|^{2}\right)^{\frac{N}{2}}\left|\frac{\left(\hat{D}^{(m)} f\right)(z)-\left(\hat{D}^{(m)} f\right)(w)}{z-w}\right|=0
$$

for all multi-index with $|m|=N-1$.

\section{The Besov space for pluriharmonic mappings}

In order to state and prove our next result, we need the following lemmas.

Lemma 5 Let $f \in \mathcal{P}\left(\mathbb{B}^{n}\right)$. Then $f \in \mathcal{B}_{p}$ if and only if

$$
\sup _{z \in \mathbb{B}^{n}}\left(1-|z|^{2}\right)^{N+1}\left(\left|\nabla\left(\hat{D}^{(m)} f\right)\right|+\left|\bar{\nabla}\left(\hat{D}^{(m)} f\right)\right|\right) \in L^{p}\left(\mathbb{B}^{n}, d \lambda\right)
$$

for all values of the multi-index $m$ with $|m|=N$, and $p(N+1) \geq n$.

Proof This follows from [12], Theorem 6.1.

Lemma 6 Let $h$ be holomorphic in $\mathbb{B}^{n}$ and $0<r<1$. Then there exist constants $K>0$, $r<r^{\prime}<1$ such that

$$
\sup _{z \in \mathbb{B}^{n}, \rho(z, w)<r, z \neq w}\left|\frac{h(z)-h(w)}{z-w}\right| \leq K \int_{E\left(z, r^{\prime}\right)}|\nabla h(u)| d \lambda(u) .
$$

Proof By the subharmonicity and Lemma 1 , for each $w \in \mathbb{B}^{n}$, we have

$$
\begin{aligned}
\sup _{z \in \mathbb{B}^{n}, \rho(z, w)<r, z \neq w}\left|\frac{h(z)-h(w)}{z-w}\right| & \leq C \sup _{\zeta \in E(z, r)}|\nabla h(\zeta)| \\
& \leq \frac{C}{\left|E\left(z, r^{\prime}\right)\right|} \int_{E\left(z, r^{\prime}\right)}|\nabla h(\zeta)| d v(\zeta) \\
& \leq K \int_{E\left(z, r^{\prime}\right)}|\nabla h(\zeta)| d \lambda(\zeta)
\end{aligned}
$$

for some $r^{\prime}>r$.

Theorem 4 Let $f \in \mathcal{P}\left(\mathbb{B}^{n}\right), N \geq 0$ be an integer and $0<r<1$. Then $f \in \mathcal{B}_{p}$ if and only if

$$
K_{f}=\int_{\mathbb{B}^{n}}\left(\sup _{z \in \mathbb{B}^{n}, \rho(z, w)<r, z \neq w} \frac{\left(1-|z|^{2}\right)^{\alpha}\left(1-|w|^{2}\right)^{\beta}\left|\hat{D}^{(m)} f(z)-\hat{D}^{(m)} f(w)\right|}{|z-w|}\right)^{p} d \lambda(z)<\infty
$$

for all values of the multi-index $m$ with $|m|=N$, where $\alpha+\beta=N+1$, and $p(N+1) \geq n$. 
Proof Let $f=h+\bar{g} \in \mathcal{P}\left(\mathbb{B}^{n}\right)$. Suppose that

$$
\int_{\mathbb{B}^{n}}\left(\sup _{z \in \mathbb{B}^{n}, \rho(z, w)<r, z \neq w} \frac{\left(1-\left|z^{2}\right|\right)^{\alpha}\left(1-\left|w^{2}\right|\right)^{\beta}\left|\hat{D}^{(m)} f(z)-\hat{D}^{(m)} f(w)\right|}{|z-w|}\right)^{p} d \lambda(z)<\infty .
$$

Let

$$
L_{f}(z)=\lim _{z \rightarrow w} \sup \frac{\left(1-|z|^{2}\right)^{\alpha}\left(1-|w|^{2}\right)^{\beta}\left|\hat{D}^{(m)} f(z)-\hat{D}^{(m)} f(w)\right|}{|z-w|} .
$$

It follows from the proof of Theorem 1 that we have

$$
\left(1-|z|^{2}\right)^{N+1}\left(\left|\nabla\left(\hat{D}^{(m)} f\right)(z)\right|+\left|\bar{\nabla}\left(\hat{D}^{(m)} f\right)(z)\right|\right) \leq C L_{f}(z) .
$$

Since $L_{f}(z) \leq L_{f}$, we see that

$$
\begin{aligned}
& \int\left(1-|z|^{2}\right)^{(N+1) p}\left(\left|\nabla\left(\hat{D}^{(m)} f\right)\right|+\left|\bar{\nabla}\left(\hat{D}^{(m)} f\right)\right|\right)^{p} d \lambda(z) \\
& \quad \leq C \int_{\mathbb{B}^{n}}\left(\sup _{z \in \mathbb{B}^{n}, \rho(z, w)<r, z \neq w} \frac{\left(1-\left|z^{2}\right|\right)^{\alpha}\left(1-\left|w^{2}\right|\right)^{\beta}\left|\hat{D}^{(m)} f(z)-\hat{D}^{(m)} f(w)\right|}{|z-w|}\right)^{p} d \lambda(z),
\end{aligned}
$$

which yields $f \in \mathcal{B}_{p}$.

To prove the necessity, we suppose that $f=h+\bar{g} \in \mathcal{B}_{p}$. By Lemmas 1 and 6 , for each multi-index $m$,

$$
\begin{aligned}
L_{f} \leq & \sup _{z \in \mathbb{B}^{n}, \rho(z, w)<r, z \neq w} \frac{\left(1-|z|^{2}\right)^{\alpha}\left(1-|w|^{2}\right)^{\beta}\left(\left|\partial^{m} h(z)-\partial^{m} h(w)\right|+\left|\partial^{m} g(z)-\partial^{m} g(w)\right|\right)}{|z-w|} \\
\leq & C \sup _{z \in \mathbb{B}^{n}, \rho(z, w)<r, z \neq w} \frac{\left(1-|z|^{2}\right)^{N+1}\left|\partial^{m} h(z)-\partial^{m} h(w)\right|}{|z-w|} \\
& +C \sup _{z \in \mathbb{B}^{n}, \rho(z, w)<r, z \neq w} \frac{\left(1-|z|^{2}\right)^{N+1}\left|\partial^{m} g(z)-\partial^{m} g(w)\right|}{|z-w|} \\
\leq & C_{1} \int_{E\left(z, r^{\prime}\right)}\left(1-|u|^{2}\right)^{N+1}\left(\left|\nabla\left(\partial^{m} h\right)(u)\right|+\left|\nabla\left(\partial^{m} g\right)(u)\right|\right) d \lambda(u) .
\end{aligned}
$$

Since

$$
\int_{E\left(z, r^{\prime}\right)} d \lambda(u)<\infty
$$

by Hölder's inequality and Fubini's theorem, we can obtain

$$
\begin{aligned}
K_{f} & \leq C \int_{\mathbb{B}^{n}}\left(\int_{E\left(z, r^{\prime}\right)}\left(1-|u|^{2}\right)^{N+1}\left(\left|\nabla\left(\partial^{m} h\right)(u)\right|+\left|\nabla\left(\partial^{m} g\right)(u)\right|\right) d \lambda(u)\right)^{p} d \lambda(z) \\
& \leq C \int_{\mathbb{B}^{n}}\left(\int_{E\left(z, r^{\prime}\right)}\left(1-|u|^{2}\right)^{(N+1) p}\left(\left|\nabla\left(\partial^{m} h\right)(u)\right|+\left|\nabla\left(\partial^{m} g\right)(u)\right|\right)^{p} d \lambda(u)\right) d \lambda(z) \\
& \leq C^{\prime} \int_{\mathbb{B}^{n}}\left(1-|u|^{2}\right)^{(N+1) p}\left(\left|\nabla\left(\partial^{m} h\right)(u)\right|+\left|\nabla\left(\partial^{m} g\right)(u)\right|\right)^{p} d \lambda(u) .
\end{aligned}
$$

It follows from Lemma 5 that $K_{f}$ is bounded. This completes the proof. 
Competing interests

The authors declare that they have no competing interests.

Authors' contributions

Both authors contributed equally to the writing of this paper. Both authors read and approved the final manuscript.

\section{Author details}

'Department of Mathematics, Shaoxing University, Shaoxing, Zhejiang 312000, People's Republic of China. ${ }^{2}$ School of Mathematics and Physics, University of South China, Hengyang, Hunan 421001, People's Republic of China.

\section{Acknowledgements}

The authors heartily thank the referee for a careful reading of this paper as well as for many helpful comments and suggestions. The research was partly supported by program for NSF of China (Nos. 11501374, 11501284), NSFs of Zhejiang (No. LQ14A010006) and Hunan (No. 2015JJ6095).

Received: 1 April 2015 Accepted: 29 October 2015 Published online: 14 November 2015

\section{References}

1. Colonna, F: The Bloch constant of bounded harmonic mappings. Indiana Univ. Math. J. 38, 829-840 (1989)

2. Yoneda, R: The harmonic Bloch and Besov spaces by an oscillation. Proc. Edinb. Math. Soc. 45, 229-239 (2002)

3. Chen, S, Ponnusamy, S, Rasila, A: On characterizations of Bloch-type, Hardy-type and Lipschitz-type spaces. Math. Z. 279, 163-183 (2015)

4. Jevtić, M, Pavlović, M: On $\mathscr{M}$-harmonic Bloch space. Proc. Am. Math. Soc. 123, 1385-1392 (1995)

5. Ren, G, Kähler, U: Weighted Lipschitz continuity and harmonic Bloch and Besov spaces in the real unit ball. Proc. Edinb. Math. Soc. 48, 743-755 (2005)

6. Rudin, W: Function Theory in the Unit Ball of $\mathbb{C}^{n}$. Springer, New York (1980)

7. Zhao, R: A characterization of Bloch-type spaces on the unit ball of $\mathbb{C}^{n}$. J. Math. Anal. Appl. 330, 291-297 (2007)

8. Chen, S, Wang, X: On harmonic Bloch spaces in the unit ball of $\mathbb{C}^{n}$. Bull. Aust. Math. Soc. 84, 67-78 (2011)

9. Li, S: Characterizations of Besov spaces in the unit ball. Bull. Korean Math. Soc. 49, 89-98 (2012)

10. Li, S, Wulan, H: Characterizations of $\alpha$-Bloch spaces on the unit ball. J. Math. Anal. Appl. 337, 880-887 (2008)

11. Ren, G, Tu, C: Bloch spaces in the unit ball of $\mathbb{C}^{n}$. Proc. Am. Math. Soc. 133, 719-726 (2005)

12. Zhu, K: Spaces of Holomorphic Functions in the Unit Ball. Springer, New York (2005)

13. Chen, S, Rasila, A, Wang, X: Radial growth, Lipschitz and Dirichlet spaces on solutions to the non-homogenous Yukawa equation. Isr. J. Math. 204, 261-282 (2014)

\section{Submit your manuscript to a SpringerOpen ${ }^{\circ}$ journal and benefit from:}

- Convenient online submission

Rigorous peer review

- Immediate publication on acceptance

- Open access: articles freely available online

- High visibility within the field

- Retaining the copyright to your article 